\title{
Uniportal video-assisted thoracoscopic lung sparing tracheo-bronchial and carinal sleeve resections
}

\author{
Diego Gonzalez-Rivas ${ }^{1,2,3 \#}$, Konstantinos Marios Soultanis ${ }^{4 \#}$, Alejandro Garcia ${ }^{2}$, Kaiyun Yang ${ }^{3}$ Yue Qing $^{3}$, \\ Linhua Yie $^{3}$, Guangqiang Zhao ${ }^{3}$, Anning Chen ${ }^{3}$, Yunchao Huang ${ }^{3}$, Guangjian Li $^{3}$, Gening Jiang ${ }^{1}$ \\ ${ }^{1}$ Thoracic Surgery Department, Tongji University Affiliated Shanghai Pulmonary Hospital, Shanghai, China; ${ }^{2}$ Department of Thoracic Surgery and \\ Minimally Invasive Thoracic Surgery Unit (UCTMI), Coruña University Hospital, Coruña, Spain; ${ }^{3}$ Thoracic Surgery Department, Yunnan Cancer \\ Hospital, the 3rd Affiliated Hospital of Kunming Medical University, Kunming, China; ${ }^{4}$ Thoracic Surgery Department, Hellenic Airforce General \\ Hospital, Athens, Greece \\ Contributions: (I) Conception and design: D Gonzalez-Rivas, KM Soultanis; (II) Administrative support: D Gonzalez-Rivas, KM Soultanis; (III) \\ Provision of study materials or patients: All authors; (IV) Collection and assembly of data: D Gonzalez-Rivas, KM Soultanis; (V) Data analysis and \\ interpretation: D Gonzalez-Rivas, KM Soultanis; (VI) Manuscript writing: All authors; (VII) Final approval of manuscript: All authors. \\ \#These authors contributed equally to this work. \\ Correspondence to: Diego Gonzalez-Rivas. Thoracic Surgery Department, Tongji University Affiliated Shanghai Pulmonary Hospital, 507 Zhengmin \\ Rd., Shanghai 200433, China. Email: diego.gonzalez.rivas@sergas.es.
}

\begin{abstract}
Pathology arising from the intrathoracic portion of the trachea (distal trachea), the carina and the main bronchi is usually neoplastic and is mainly treated with surgery. Resection of the intrathoracic portion of the trachea, the carina and the main bronchi for neoplastic lesions does not necessitate lung resection and is traditionally being conducted via open surgery. Video-assisted thoracic surgery (VATS) is witnessing an exponential growth and is the treatment of choice for early-stage non-small cell lung cancer (NSCLC). The experience accumulated over the past two decades along with the introduction of reliable and ergonomic technology, has led to the expansion of its indications. In this article we provide a detailed description of lung sparing distal tracheal, carinal and main bronchi resection for primary neoplasms of the airway, without involvement of the lung, with the uniportal video-assisted technique. The chest is entered through the fourth intercostal space, mid-axillary line. Dissection of the paratracheal space anteriorly, the tracheoesophageal groove posteriorly and the subcarinal space and division of the azygos arch are essential to mobilize the distal trachea and carina. Lateral dissection should be avoided beyond the points of division of the airway, as it may hinder the blood supply to the anastomosis. Any tension to the anastomosis should be relieved by release maneuvers. Ventilation is achieved through an endobronchial catheter, inserted into the left main bronchus through which a high-frequency jet ventilation catheter can be also inserted through it. The rationale of applying a minimally invasive technique for the conduction of tracheal and carinal resections, is to exploit its advantages, namely less pain, earlier mobilization and lower morbidity. Uniportal video-assisted resections of the distal trachea, carina and the main bronchi, are safe when conducted by experienced surgical and anesthetic teams.
\end{abstract}

Keywords: Uniportal sleeve; carinal resections; sparing lung resections; trachea-bronchial sleeve; bronchoplasty; sleeve reconstruction; advanced video-assisted thoracic surgery (advanced VATS)

Submitted Dec 09, 2019. Accepted for publication Mar 25, 2020.

doi: $10.21037 /$ jtd.2020.04.05

View this article at: http://dx.doi.org/10.21037/jtd.2020.04.05 


\section{Introduction}

The intrathoracic portion of the trachea and carina has been referred to as the "last unpaired organ in the body to fall to the surgeon" (1). Concerns about poor cartilage healing, the belief that a very limited segment of the trachea could be resected, the challenges related to intraoperative ventilation during tracheal resection have been named as the main reasons for delayed progress in this particular field of surgery. Equally incremental, has been the fact that tracheal resection was seldom needed, since the rarity of tracheal tumors and the absence of mechanical ventilatory support, responsible for benign post-intubation stenosis, had as a result tracheal resections and end-to-end anastomosis being rarely performed (2). Firm evidence confirming cartilage healing were available long before Miura, Grillo and Salassa described in detail the blood supply of the trachea, directly linking the surgical technique with the success of a tracheal resection and reconstruction but were forgotten and resurfaced following the end of the Second World War (1,3-6). Anesthesia also evolved, successfully solving the problem of control of the airway and effective ventilation, without compromising the surgical exposure of the trachea, by means of cross-field ventilation and high-frequency jet ventilation $(7,8)$. The development of release maneuvers and the implementation of neck flexion during and after reconstruction of the trachea, dissolved the " $2 \mathrm{~cm}$ rule", the belief that tracheal resections longer than 2 centimeters were not feasible, and allowed the resection of segments as long as half the length of the trachea (9).

The most frequent indications for elective tracheal resection and reconstruction, include benign tumors and strictures, often attributed to prolonged intubation (postintubation strictures) and malignancies. Surgical resection of these tumors is the mainstay of treatment. Particularly the distal third of the trachea and carina are the sites of primary tracheal tumors, usually malignant, or may be infiltrated by lung cancer. Resection of tumors arising at or involving the distal part of the trachea and/or the carina is typically performed via a right posterolateral thoracotomy, through the $4^{\text {th }}$ or $5^{\text {th }}$ intercostal space, or through the bed of the resected $4^{\text {th }}$ rib (9). A posterolateral thoracotomy provides an excellent exposure of the posterior mediastinum, where the lower trachea, carina and main bronchi are situated (10). However, as experience over video-assisted thoracic surgery (VATS) grows, this traditional approach is being challenged. During the years following McKenna's et al. first report of VATS sleeve lobectomy, VATS has witnessed an unprecedented spread both in geographical terms and indications, while at the same time the technique is being constantly modified and improved towards becoming as less invasive as possible, from 3-4 ports to the uniportal technique (11-16). Reports of video-assisted tracheal resections are promising short and mid-term results comparable to open resections $(17,18)$. It seems that after a "slow start", at the mid-twentieth century and the establishment of the principles of tracheal surgery in the 70s, the era of VATS resection of the intrathoracic portion of the trachea and the carina has dawned, again with some lag compared to other intrathoracic organs such as the lung, the esophagus and the mediastinum. This of course, should not be interpreted as a shift in surgical principles of tracheal resections, but rather as an attempt to improve their outcomes. VATS has proven superior to open surgery in terms of pain, postoperative length of hospital stay, morbidity, quality of life, immunosuppression and possibly long-term survival in lung cancer patients and is now the treatment of choice for early-stage non-small cell lung cancer (NSCLC) (19-24). Particularly, the uniportal VATS technique has gained great momentum since it was first implemented for major, anatomic pulmonary resections in 2010 (25) and for complex procedures such as sleeve resections in $2012(14,15)$. There are evidence that it is associated with less postoperative pain in the early postoperative period compared to multiport techniques, probably due to less torqueing of the thoracoscopic instruments and the fact that intercostal nerve injury is limited to the nerve adjacent to the incision (26).

In this article, we describe the case of a carinal resection combined with right upper lobectomy for a locally advanced adenoid cystic carcinoma (ACC), a right main bronchus resection for an endobronchial adenocarcinoma located near the carina, a case of resection and reconstruction of the secondary carina due to an endobronchial squamous cell carcinoma attempted via a hilar anterior approach and a case of a left main bronchus resection and reconstruction of the secondary carina due to a mucoepidermoid carcinoma, performed via a posterior approach. All cases were conducted via the uniportal VATS technique and were discharged from the hospital few days later after an uneventful hospitalization.

\section{Preoperative planning and evaluation}

Preoperative planning and evaluation include an attempt for obtaining a preoperative tissue diagnosis, staging of the 


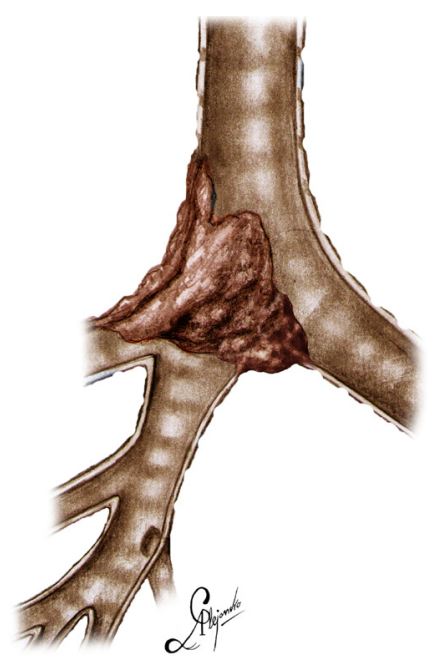

Figure 1 Graphical depiction of the carinal tumor.

tumor and a physiologic evaluation of the patient.

A computed tomography (CT) of the chest with intravenous contrast, is indicated for the identification of the tumor's location, its extension into the parenchyma and its relationship with surrounding structures (esophagus, lung). Information regarding regional lymph nodes, as well concurrent atelectasis in case of an endobronchial tumor, may also be obtained.

Further characterization and staging of the tumor require a positron emission tomography (PET) scan. An increased standard uptake value (SUV) of the lesion and/ or the regional lymph nodes may indicate a malignancy and guide attempts for tissue diagnosis and invasive lymph node assessment.

Tissue diagnosis and staging may be obtained via bronchoscopy. Rigid bronchoscopy can be utilized to relieve obstruction prior to surgery (27). Bronchoscopy in combination with chest CT are used to determine the extent of the trachea and/or bronchi needed to be resected. This information is crucial in determining the operability of the tumor, in an otherwise local, non-metastatic lesion.

For the physiologic evaluation of the patient a predicted postoperative forced expiratory capacity in the first second (FEV1) should be initially evaluated. If impaired, a predictive postoperative carbon monoxide diffusion capacity of the lung and/or a cardiopulmonary exercise test (CPET) should be obtained (28). Although this assessment is proposed for curative intent pulmonary resections and not for lung sparing procedures, one should keep in mind that when attempting a lung sparing carinal or main bronchus resection, there is a possibility that the attempt may fail and the lung lost Cardiac function should be assessed with an electrocardiogram and depending on the patient's medical history, with further testing (e.g., echocardiography).

\section{Case 1: carinal resection and right upper lobectomy}

The operation was performed for a locally advanced ACC, infiltrating the distal third of the trachea and carina extending into the right upper lobe bronchus and intermedius bronchus involving also the left main bronchus (Figure 1), (Video 1).

The patient's left main bronchus was intubated with a long endobronchial tube, through which a catheter was passed for the implementation of high frequency jet ventilation (HFJV) during the reconstruction (when the tube is withdrawn). The endobronchial tube was repositioned in the left main bronchus once its anterior wall was sutured to that of the trachea and the HFJV catheter withdrawn. Should the tumor had not been extending to the bronchus for the upper lobe, the lobe would have been spared. As it was intraoperatively revealed by the frozen section examination of the resected specimen, the bronchus intermedius was also infiltrated. The infiltration of the bronchus intermedius was not indicated by the preoperative chest CT. The inferior pulmonary ligament was released before the reconstruction, however after the reconstruction there was a need to further relieve the tension of the anastomosis and a typical intrapericardial release was performed (29).

The patient was positioned in the left decubitus position. The incision was sited at the fourth intercostal space, at the level of the middle axillary line. For an upper lobectomy siting the incision between the anterior and the middle axillary line and even more anteriorly is more convenient, because an upper lobectomy can be performed without extensive dissection in the posterior mediastinal compartment and the dissection of station 7 is not hindered. But for a carinal resection, unimpaired visualization of the posterior mediastinum is necessary, because the distal trachea and the left main bronchus have to be adequately dissected before resection. The camera is always at the posterior end of the incision, providing a view similar to that obtained during open surgery and sparing the rest of the space for at least two instruments, the sucker and an energy device or dissecting instrument which are handled by the surgeon. The placement of a wound protector not only protects against tumor seeding, but also adds extra space for 


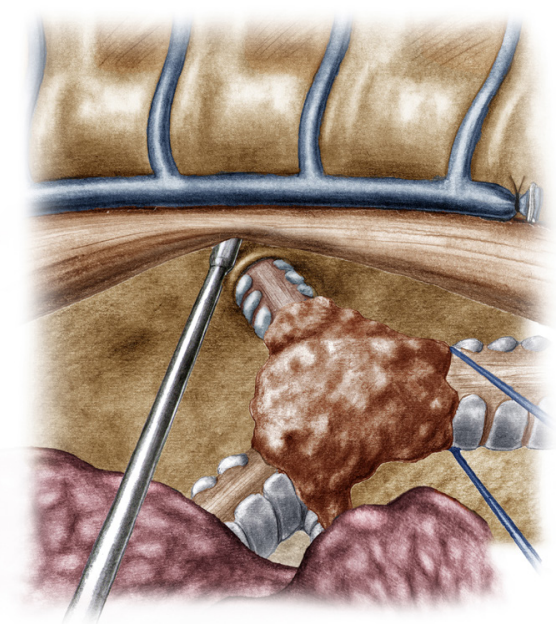

Figure 2 View of the distal trachea, the carina (infiltrated by the tumor), the right and left main bronchus.

the insertion of a fourth instrument, a lung grasper handled by the assistant and used to maintain the lung retracted. The table was slightly tilted anteriorly to displace the lung anteriorly and expose the posterior mediastinum. Such a maneuver may sometimes eliminate the need for grasping and retracting the lung.

The operation began with dissection along the inferior rim of the azygos vein arch, in an attempt to gain access and dissect the paratracheal space. The paratracheal lymph nodes (stations $2 \mathrm{R}$ and $4 \mathrm{R}$ ) were harvested and the anterior cartilaginous wall of the trachea exposed almost up to the level of the innominate artery. Lateral dissection from both sides of the trachea was avoided in order not to disrupt the longitudinal arterial network responsible for the blood supply of the tracheal cartilages. As the dissection moved posteriorly, towards the right side of the trachea, the right vagus nerve was found thickened and firmly adjacent to the tracheal wall, indicating infiltration by the tumor and had to be transected. After confirming infiltration of the azygos vein arch by the tumor, the dissection was continued across the tracheoesophageal groove, (the esophagus was identified), up to the superior intercostal vein which was ligated with polymer clips and disconnected from the azygos arch with the energy device. Subsequently the azygos vein was divided with an endostapler both at its junction with the superior vena cava anteriorly and posteriorly. Subsequently separation of the posterior tracheal wall from the esophagus was carried out by carefully dissecting along the tracheoesophageal groove, by means of blunt (sucker) and sharp dissection (energy device), taking care not to injure the posterior wall of the trachea and the esophagus. It relies upon the surgeon's preference to ask for a boogie to be inserted in the esophagus, in order to make this dissection safer. The role of the sucker in such a dissection is paramount (as is generally in the uniportal VATS instrumentation philosophy), as not only does it clear the field of blood, but also provides the surgeon with a tactile sense of the structures he/she is dissecting. This dissection is an example of the uniportal VATS bimanual instrumentation, with which the two instruments handled by the surgeon are simultaneously engaged in the dissection of a structure under the view of the 30 degrees, $10 \mathrm{~mm}$ scope. The dissection along the tracheoesophageal groove was carried out beyond the posterior wall of the left main bronchus, which was identified, up to the subcarinal space, which was dissected (removal of station 7 lymph nodes), clearly revealing the carina, infiltrated by the tumor, and the inferior rim and posterior wall of the left main bronchus (Figure 2). Dissection was now directed to the hilum. Hilar lymph nodes (10R), just below the level of azygos arch, were removed and the truncus anterior transected revealing the anterior wall of the right main bronchus, which was then separated from the underlying right pulmonary artery (PA). The truncus was transected with a curved tip stapler, which is very useful in cases where the structures are closely and firmly adjacent to one another, as in this case. At this point, became evident that the right upper lobe bronchus could not be spared, and a lobectomy was decided. The superior pulmonary vein was then dissected, the middle lobe vein identified, and the distal aspect of the transverse fissure was dissected in order to facilitate the dissection of the upper lobe vein, which was transected shortly after. After the identification of the middle lobe artery, the fissure was completely dissected, revealing two arterial branches for the segment 2, which were stapled. The inferior pulmonary ligament was released. Then the distal trachea, which was previously dissected, was encircled with a vessel loop and retracted upwards and anteriorly facilitating the separation of the right main bronchus and the bronchus intermedius from the PA and the division of the bronchus intermedius (Figures 3,4). A full thickness, 3/0 prolene stay suture (figure of eight) was applied to the trachea proximally to the lesion and the bronchus intermedius was divided with scissors (and the bronchial artery with energy). The upper lobe bronchus was cut with scissors and the upper lobe retrieved in a glove. This was done in order to clear the operating field and facilitate the carinal resection and reconstruction. 


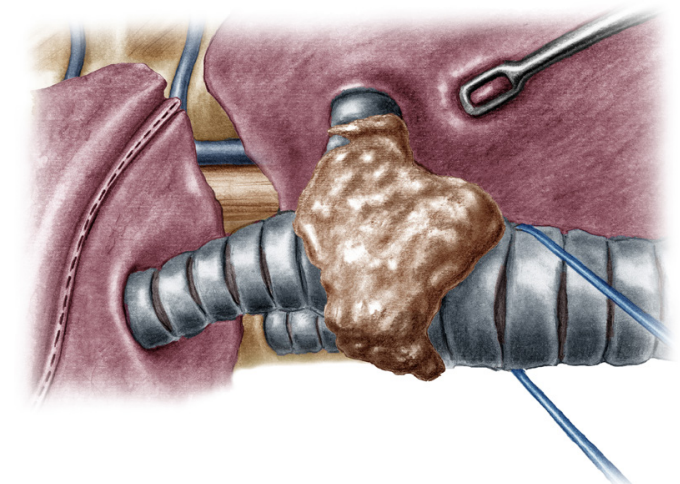

Figure 3 Traction of the trachea upwards and towards the surgeon to facilitate the division of the bronchus intermedius.

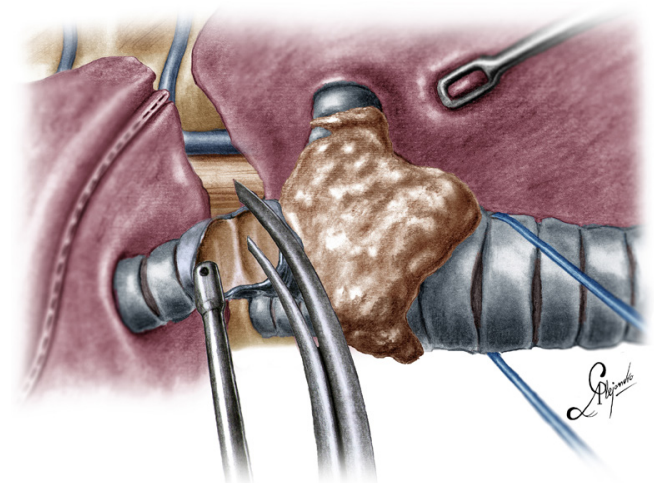

Figure 4 Division of the bronchus intermedius.

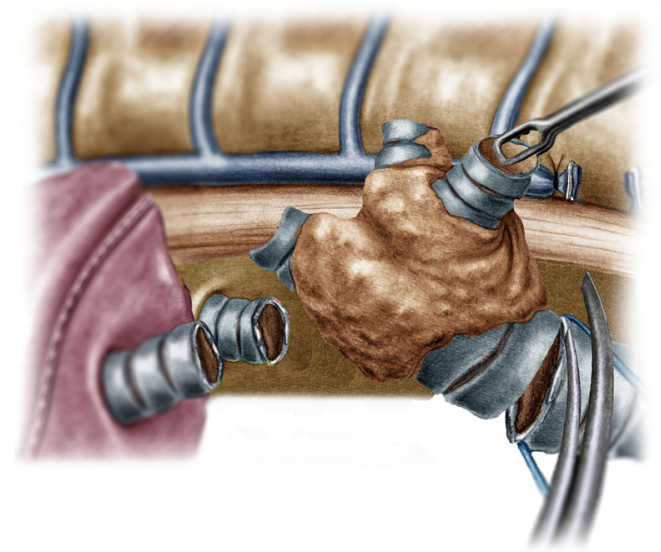

Figure 5 Division of the distal trachea.

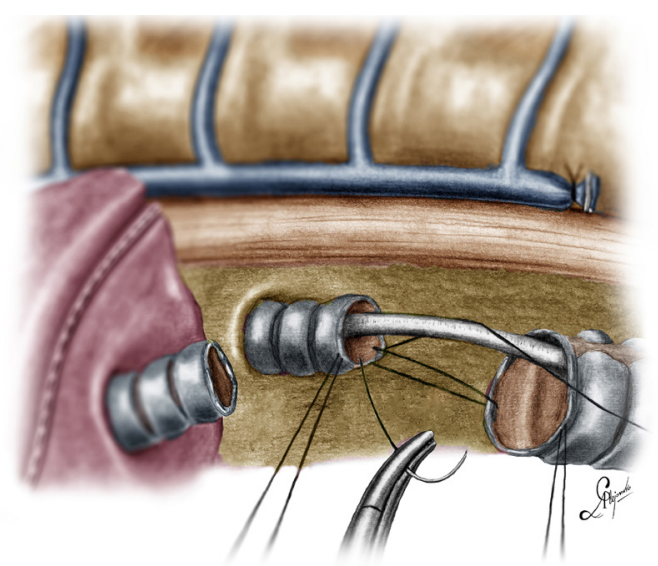

Figure 6 Initial suture between the distal trachea and the left main bronchus. Note the HFJV catheter. HFJV, high frequency jet ventilation.

A full thickness stay suture was placed in the inferior rim of the left main bronchus and the trachea was cut with scissors proximally to the lesion revealing the endobronchial tube running into the left main bronchus. The cut was extended to the left main bronchus (Figure 5). The endotracheal tube was pulled back and HFJV commenced. The reconstruction started by suturing the left main bronchus to the trachea. The first stich ( $3 / 0$ prolene, two needles), was placed insideout at the cartilaginous wall of the trachea, very close to the junction with the membranous wall, and connected accordingly, outside-in with the cartilaginous wall of the left main bronchus, close to its junction with the membranous portion and continued connecting the anterior wall of the trachea with that of the left main bronchus (approximately half of their circumference) (Figure 6). The suturing continued with the other needle connecting a portion of the membranous part of the trachea to a portion of that of the left main bronchus. This suture was tied to a separate interrupted suture that was inserted for this reason. At this point, approximately half of the anterior and posterior wall of the trachea were tied to the anterior and posterior wall of the left main bronchus. A third double needle, 3/0 prolene was used to tie the remaining half of the anterior wall of the left main to the adjacent half of the anterior wall of the bronchus intermedius (Figure 7). This suture was tied to the suture used to connect the anterior wall of the 


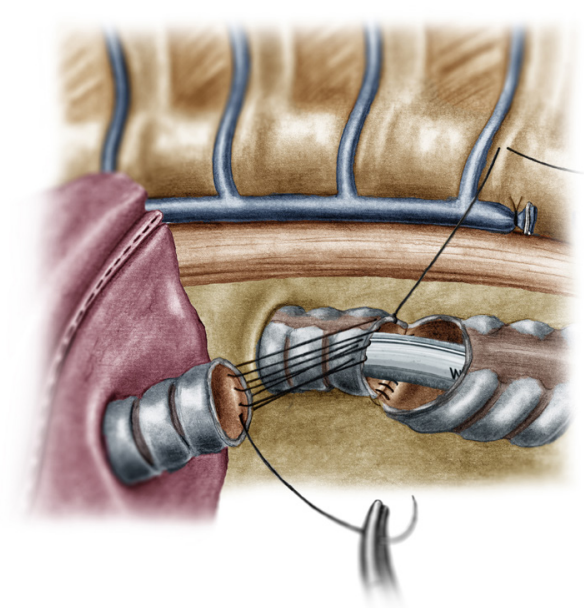

Figure 7 Reconstruction of the medial wall of the neo-carina (median walls of left main bronchus and bronchus intermedius).

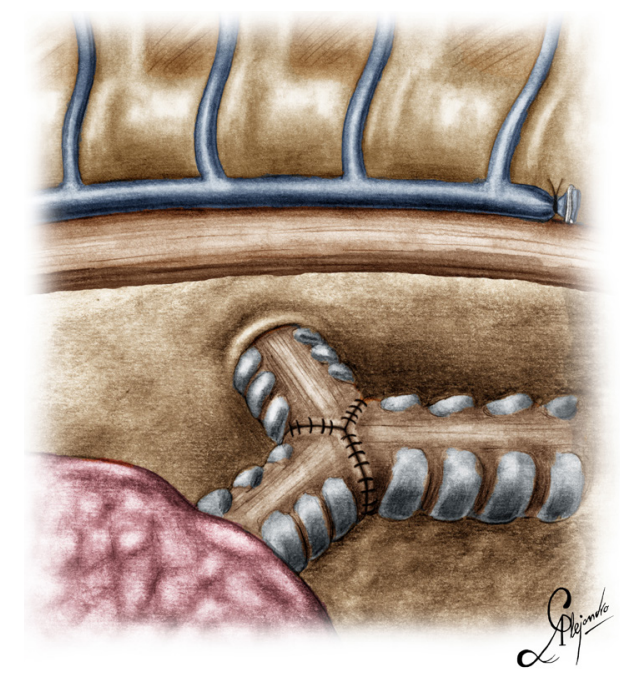

Figure 8 Graphic depiction of the neo-carina after the reconstruction.

trachea to the anterior wall of the left main bronchus. The other needle of this suture was used to tie the remaining half of the posterior wall of the left main to the adjacent half of the posterior wall of the bronchus intermedius and the remaining half of the posterior wall of the trachea. This suture was tied to a fourth $3 / 0$ prolene suture which approximated the remaining portions of the anterior walls of the trachea and the bronchus intermedius, completing the reconstruction (Figure 8) (Video 1). Following the reconstruction, tension of the suture line was evenly distributed using a hook and a typical intrapericardial release was added to ensure a tension free anastomosis. Air leak test was negative. A 28 -Fr chest tube was placed and the chest was closed.

\section{Case 2: uniportal VATS lung sparing right main bronchus resection}

This was a case of a lung sparing right main bronchus resection for an endobronchial adenocarcinoma situated at the entrance of the bronchus, close to the carina. The patient had a history of tuberculosis, so the lung was almost completely fused to the parietal pleura with dense and firm adhesions. A typical left double lumen endotracheal tube was used to ventilate the patient and block the right lung and the patient was positioned to the left decubitus position.

The incision was sited at the fourth intercostal space, at the middle axillary line, For the resection of the right main bronchus and reapproximation of the distal bronchial tree to the carina an unimpaired visualization and access to the posterior mediastinum are needed. This is because the distal third of the trachea, the carina and the left main bronchus have to be dissected and mobilized. As demonstrated in the previous case, the mobilization of the intrathoracic trachea is accomplished with the division of the azygos vein arch and the dissection of the paratracheal space. The dissection of the paratracheal space does not impair the tracheal blood supply, as long as it does not extend along either sides of the trachea. The carina and the left main bronchus (posterior wall) are exposed following careful dissection along the tracheoesophageal groove, which leads to the subcarinal space. The removal of station 7 lymph nodes greatly facilitates the mobilization of the carina and the left main bronchus. The distal trachea was encircled with a tape and retracted towards the surgeon and the right main bronchus was incised circumferentially removing the affected part on healthy tissue (histologically confirmed via a frozen section examination). Because of the short length of the right main bronchus, what was left was actually the entrance of the right upper bronchus and the bronchus intermedius. Reapproximation of the bronchial tree was done with a double needle, $3 / 0$ continuous, prolene suture. The first stich was placed inside-out at the cartilaginous wall of the trachea, very close to the junction with the membranous wall and connected outside-in at the cartilaginous wall of the right upper bronchus and continued all the way connecting the anterior wall of the trachea to the anterior walls of the 
A

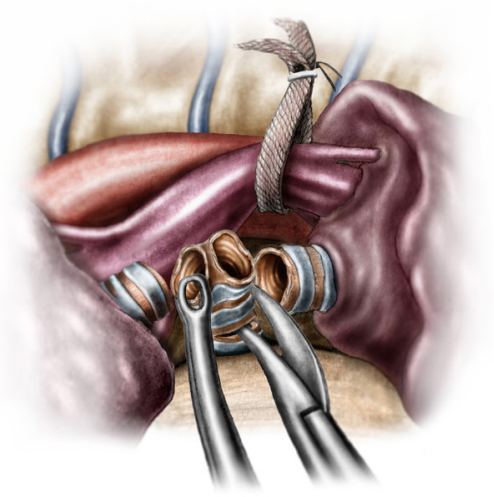

B

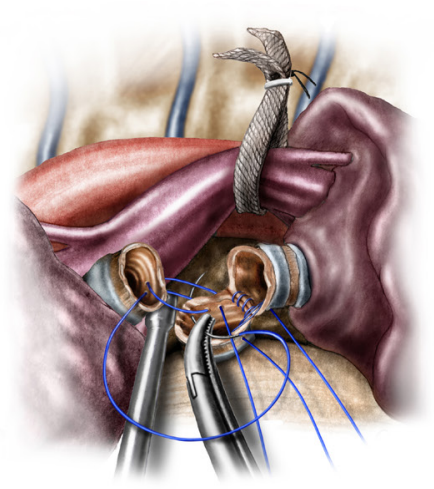

C

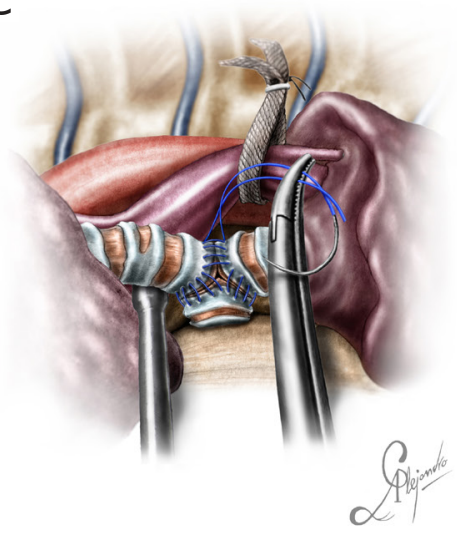

Figure 9 The upper and lower lobe bronchus and the secondary carina have been fully mobilized. The pulmonary artery is suspended via a tape from the chest wall to allow unobstructed reconstruction of a secondary neo-carina. (A) Resection of the secondary carina; (B) reconstruction of the secondary carina; (C) the upper and lower lobe bronchus have been re-approximated and a neo-secondary carina reconstructed.. The posterolateral wall of the lower bronchus is anastomosed first to lateral wall of left main bronchus and then is sutured to the posterolateral wall of the left upper bronchus. Note the PA retracted providing space for the suturing. The secondary carinal anastomosis after the reconstruction. PA, pulmonary artery.

right upper bronchus and the bronchus intermediate. The posterior wall of the anastomosis was sutured with the other needle of the $3 / 0$ prolene suture. The two sutures were tied together at the side of the trachea (Video 2). Following the negative air leak test, a 28-Fr tube was left before closing the chest.

\section{Case 3: uniportal VATS resection and reconstruction of the secondary carina}

This was a case of an endobronchial squamous cell carcinoma, located at the bifurcation of the left main bronchus (secondary carina). The patient was positioned in the right decubitus position, following endotracheal intubation with a right double lumen endotracheal tube. A left endotracheal tube in this case would have made the mobilization and manipulation of the left main bronchus during the reconstruction difficult.

The bifurcation of the left main bronchus was approached form the anterior. Initially the posterior aspect of the fissure was opened, and the lingular artery identified, exposed and transected. The lingular artery had to be sacrificed in order to obtain access to the secondary carina. Station 11 lymph nodes (at the bifurcation) were sampled and the anterior portion of the fissure was also completed. At this point the secondary carina was completely exposed just below the PA. The lower bronchus was freed from the overlying arterial trunk for the lower lobe via blunt bimanual dissection with the "snake" forceps and the sucker from the anterior and the subcarinal space was cleared. The pulmonary ligament was released, and the space between the lower bronchus and the inferior pulmonary vein was dissected. The lower lobe artery was encircled with a tape and suspended, and the upper and lower bronchus were encircled with a silk suture and retracted towards the surgeon. The space between the anterior wall of the main and left upper bronchus and the superior pulmonary vein was dissected. At this point the structures of interest, the left main bronchus, the upper lobe bronchus, the secondary carina, the lower bronchus and the PA for the lower lobe, were all fully mobilized. The lower lobe bronchus was cut first and disconnected at the level of the bifurcation, followed by the upper lobe bronchus. The secondary carina was grasped with a forceps and pulled towards the surgeon allowing for the division of the main bronchus proximal to the lesion (Figure 9A). The reconstruction was initiated by suturing the lower bronchus to the main bronchus with a double needle, $3 / 0$ prolene and continued in exactly the same fashion described in case 1 , until a neo-secondary carina was formed (Figure 9B,C). Air leak test was negative, a $28-\mathrm{Fr}$ chest tube was placed and the chest closed (Video 3). 


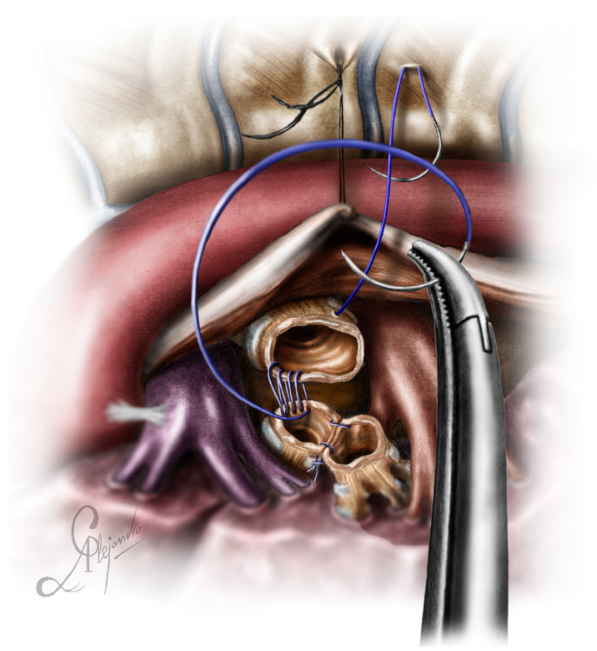

Figure 10 The posterior mediastinal pleura and the underlying esophagus are suspended to facilitate the construction of a neocarina.

\section{Case 4: uniportal VATS lung sparing left main bronchus resection and reconstruction of the secondary carina}

In this case, the left main bronchus was resected due to an endobronchial mucoepidermoid carcinoma arising from the membranous wall just before the bifurcation. Circumferential resections of a portion of the left main bronchus close to the secondary carina, usually require reconstruction of the secondary carina. This case required extensive dissection all around the hilum and the inferior pulmonary vein, after which all the structures were fully mobilized. The patient was intubated with a right double lumen endotracheal tube and positioned in the right decubitus position.

Dissection started at the posterior mediastinum by incising the pleura just anterior to the vagus nerve and proceeded caudally up to the outer curvature of the PA. The subcarinal and subaortic space, as well as the fissure between segments $1+2 \mathrm{c}$ and 6 were also dissected. At the end of the posterior dissection all structures where clearly visible at their full length: The outer curvature of the PA, the membranous wall of the left main bronchus from the secondary carina up to the carina, the posterior pericardium up to the esophagus and the inferior pulmonary vein. The dissection was resumed anteriorly, completely freeing the $\mathrm{PA}$, the superior pulmonary vein and the anterior wall of the secondary carina. Traction sutures were used to suspend the incised posterior mediastinal pleura with the esophagus (Figure 10), and reflect the upper lobe anteriorly, providing full exposure of the main bronchus and its bifurcation (from the posterior). Once dissected, the main bronchus was encircled with a suture, lifted and pulled towards the surgeon. This maneuver facilitated the first, distal cut, at the level of the bifurcation. After the cut, the lumen of the bronchus was inspected, in order to locate the tumor and grasped facilitating the proximal division of the bronchus. A 3/0 double needle, prolene suture was used for the reconstruction. The stumps of the upper and lower bronchus were reconnected and then reattached to the main bronchus (Video 4). Air leak test was negative, and the chest was closed after placement of a 28 -Fr chest tube.

\section{Distal tracheal resection}

For a distal tracheal resection, access is achieved via a posteriorly placed incision in the $3^{\text {rd }}$ or $4^{\text {th }}$ intercostal space. The paratracheal space has to be dissected, as well as the tracheoesophageal groove, as described in case 1. Care must be taken to avoid lateral dissection, as this may devascularize the anastomosis. Lateral dissection should not extend beyond the site of the proximal cut. The azygos vein can be dissected if better exposure is required. The vagus nerve can be mobilized and spared if it is not involved. It is better the cut be done under bronchoscopic guidance to ensure tumor free margins (which have to be confirmed histologically with a frozen section biopsy) and avoid excessive resection which could jeopardize the anastomosis. Both main bronchi have to be mobilized as well. This will facilitate the anastomosis. Applying traction to the trachea via a vessel loop or tape significantly helps the dissection. Stay sutures are also a good strategy to prevent the distal airway from sliding back, although if the carina is preserved this does not usually happens (Figure 11). The reconstruction is done via a double needle, 3/0 prolene (or PDS) suture as indicated in Figure 12 (one running suture, one thread with two needles).

\section{Discussion}

Primary tumors of the trachea, of which squamous cell carcinoma and ACC are the commonest are the main indications for a carinal resection (30). Both are treated surgically. The distal bronchial tree may also be affected. Surgical therapy is significantly superior compared to palliative therapy regarding survival, provided the disease is not metastatic and the resection is complete (31). When these tumors are contained into the lumen without invading 


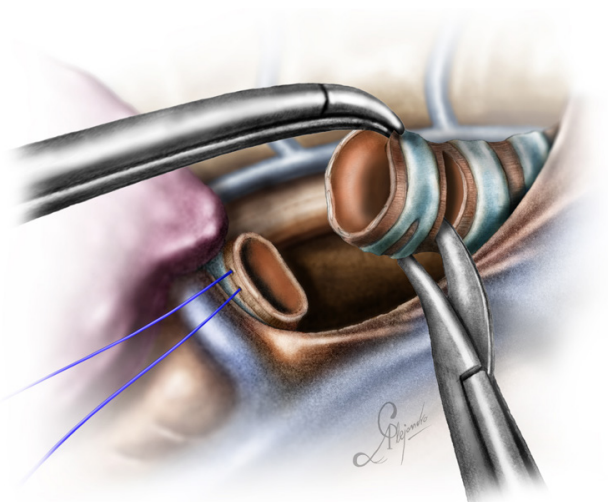

Figure 11 Cutting the trachea proximally. Note the stay sutures placed at the distal part airway.

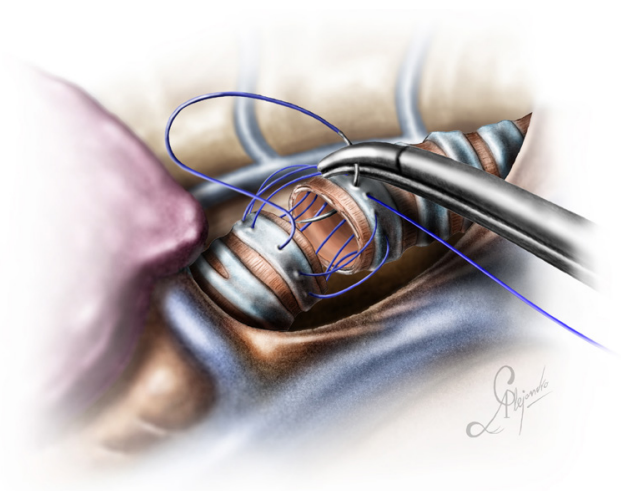

Figure 12 End-to-end anastomosis of the proximal and distal portion of the trachea.

the parenchyma, a lung sparing resection is recommended. Grillo stated that the initial problem the surgeon has to solve before attempting the resection, is to decide upon the resectability of the tumor (32). Because a reliable interposition graft is yet to be developed (33), there is a limit over the length of the trachea that can safely be removed. Techniques and strategies have been described, which can make resection of long segments feasible (9). Regarding the intrathoracic portion of the trachea and the carina, hilar dissection and release of the pulmonary ligament allow the resection of a segment as long as almost $4 \mathrm{~cm}$ (9). Intrapericardial dissection can add up to $1 \mathrm{~cm}$ (9). The laryngeal release maneuvers do not impact the mobility of the lower trachea, so one should rely upon the intrathoracic dissection for the resection of the lower trachea and carina (34).

These resections and the subsequent reconstructions are demanding even when they are performed via open surgery. Even in expert hands complications may be disastrous. In their 2004 analysis of a series of more than 900 patients, Wright et al. did not include patients with carinal resection as they believed that such patients "present a unique complication profile" (35). Nonetheless they revealed several factors predisposing to them, the most important being the length of the resected segment, diabetes mellitus and re-operation. Regarding the length of the resected segment Grillo demonstrated in cadavers the exponential rise of the anastomotic tension as the length of the segment to be resected increases and suggested any resection more than $4 \mathrm{~cm}$ to be accompanied with a release procedure $(35,36)$. Mortality after carinal resection varies from to $7.2-29 \%$ in various series reported (37). However, more contemporary series, demonstrate improved mortality reflecting the advances in patient selection, operative skills and postoperative care. Mortality after lung sparing carinal resections is attributed to anastomotic related complications and is generally lower compared to carinal pneumonectomy (38). A tissue flap is recommended for covering the anastomosis $(39,40)$.

Continuous or interrupted sutures, by now it has become clear that there is no significant difference in the outcome between the two, meaning that anastomotic related complications such as dehiscence of the suture line, bronchopleural fistulas and late stenosis are similar between the two techniques as long a non-irritable material is used and the anastomosis is tension free (40-42). Goldstraw's team was one of the first to apply a continuous suture for the bronchial reconstruction after a sleeve lobectomy (43).

Surgeons operating on the trachea and main bronchi, should possess deep knowledge of the blood supply of the trachea. The cervical portion of the trachea is supplied by the inferior thyroid artery, which via three tracheoesophageal tributaries finally produces a longitudinal anastomotic network which courses at either side of the tracheal wall. From this network intercartillaginous arteries are formed which connect with the opposite ones and form a network which supplies the mucosa and through which the cartilaginous rings are also supplied. A separate network feeds the membranous wall, but at the mucosal and submucosal level the two networks interconnect. The most caudal of these branches, anastomoses with the longitudinal network of the intrathoracic trachea, which arises from a number of different arteries, namely the innominatesubclavian artery, the supreme intercostal artery and the right internal thoracic artery. This means that the blood supply of the thoracic trachea is more variable than that of the cervical trachea. The blood supply of the distal trachea is 
more constant and arises from the bronchial arteries which may also vary in number. The bronchial arteries also interconnect with the arterial network of the intrathoracic trachea (6). This particular architecture of the arterial network of the trachea, dictates the avoidance of any lateral dissection extending further than the proximal and distal borders of the segment to be resected. Surgeons should always apply this fundamental principle regardless their technique, open or VATS.

Unlike a, for example, VATS right upper sleeve lobectomy, where the proximal and distal bronchial stumps are aligned significantly facilitating the reconstruction, the reconstructions described in the aforementioned cases are more complex. Firstly, the stumps involved are three, instead of two. Secondly there is a significant discrepancy between the caliber of the stumps especially in a case of a carinal reconstruction. Thirdly, the stumps are not aligned, but rather completely dissociated inside the pleural cavity. Their approximation requires precise suturing of the corresponding parts. To tackle the caliber discrepancy, sutures in the proximal stump are placed at a slightly larger pace than the corresponding sutures in the distal stump. Intussusception of the distal stumps into the proximal stump, which may happen during an end-to-end anastomosis (for example during a sleeve anastomosis), is not desired in a case of a carinal (primary or secondary) reconstruction. The last reason is that, while after a sleeve lobectomy the surgical specimen is removed from the field, in a lung sparing carinal and/or bronchial resection all lobes are still attached, so the field is "congested". This peculiarity is not present in open procedures. A surgeon who has not previously conducted a complete dissection of the involved and neighboring structures, will not only face potentially increased tension in the anastomosis, but also difficulty during its construction. The "congested" thoracic cavity can be also dealt with the application of traction in the surrounding structures, after they have been dissected and encircled with a tape or silk suture, as described in case 4 . Although in case 1 we described and demonstrated the division of the azygos vein (recommended in most of the cases), it is also an option to encircle it with a suture and suspend it from the chest wall. Traction sutures also eliminate the need for extra instruments. In this way adequate space is reserved for the surgeon to conveniently handle his/her instruments through the single port.

Airway management poses another challenge during carinal and bronchial resections. In case of a carinal resection cross-field ventilation or high-frequency jet ventilation, both present effective strategies during an open or VATS procedure $(7,8)$. In case of a VATS procedure an extra incision is needed for the cross-field ventilation system. Another effective strategy is the catheterization of the left main bronchus with a bronchial catheter, which resembles a double lumen endotracheal tube, from which the tracheal lumen has been removed (44). Through it, a jet ventilation catheter can be also inserted. High-frequency jet ventilation has the advantage over a cross-field tube or an endobronchial tube, that it permits unobstructed visualization and manipulation of the stumps during the reconstruction. Endobronchial intubation of the opposite bronchus (usually the left) from the beginning and a jet ventilation catheter inserted through it is the ideal strategy for uniportal VATS. During the reconstruction, the endobronchial tube is removed and repositioned after the posterior wall of the anastomosis with the trachea has been completed. Cardiopulmonary bypass in another option but is seldom needed (45). When intubation cannot be achieved preoperatively because of an obstructing lesion at the level of the carina, the lesion can either be cored out via rigid bronchoscopy and then have an endotracheal tube placed or initiate a veno-venous bypass (46). Recently a team from Guangzhou, China, reported their experience with spontaneous ventilation for VATS tracheal and carinal resections with excellent outcomes (2).

This paper and the accompanying videos demonstrate that uniportal VATS carinal and bronchial resections and reconstructions are feasible and safe in expert hands and describe the technique step by step. Li et al., Jiang et al. and Lyscov et al. have published their series of VATS tracheal and carinal resection and reconstruction with excellent and promising results $(17,45,47)$. Because what significantly increases the complexity of a VATS tracheal, carinal and bronchial reconstruction from a surgeon's point of view is the need for precise suturing, expertise can only be achieved gradually via VATS sleeve lobectomies. Some publications have stated that before attempting a VATS sleeve lobectomy, more than 200 VATS standard lobectomies and 20 open sleeve lobectomies have to be conducted to achieve optimal outcomes (13). Naturally one would ask how many VATS sleeve lobectomies and open tracheal, carinal or bronchial resections a surgeon should perform before attempting a VATS tracheal, carinal or bronchia resection. Although an answer to this question would be arbitrary, it is useful to remember that the fundamental technical principles of tracheal surgery, no lateral dissection to avoid devascularization, tension free anastomosis and disease free margins, remain exactly the same, no matter the approach, open or VATS. 


\section{Acknowledgments}

Funding: None.

\section{Footnote}

Provenance and Peer Review: This article was commissioned by the Guest Editor (Servet Bölükbas) for the series "Airway Surgery" published in fournal of Thoracic Disease. The article was sent for external peer review organized by the Guest Editor and the editorial office.

Conflicts of Interest: All authors have completed the ICMJE uniform disclosure form (available at http://dx.doi. org/10.21037/jtd.2020.04.05). The series "Airway Surgery" was commissioned by the editorial office without any funding or sponsorship. DGR serves as the unpaid editorial board member of Fournal of Thoracic Disease from Jan 2019 to Dec 2021. The other authors have no other conflicts of interest to declare.

Ethical Statement: The authors are accountable for all aspects of the work in ensuring that questions related to the accuracy or integrity of any part of the work are appropriately investigated and resolved.

Open Access Statement: This is an Open Access article distributed in accordance with the Creative Commons Attribution-NonCommercial-NoDerivs 4.0 International License (CC BY-NC-ND 4.0), which permits the noncommercial replication and distribution of the article with the strict proviso that no changes or edits are made and the original work is properly cited (including links to both the formal publication through the relevant DOI and the license). See: https://creativecommons.org/licenses/by-nc-nd/4.0/.

\section{References}

1. Belsey R. Resection and reconstruction of the intrathoracic trachea. Br J Surg 1950;38:200-5.

2. Grillo HC. Development of Tracheal Surgery. In: Grillo HC. Surgery of the trachea and bronchi. Raleigh: PMPH USA, 2004.

3. Kuster E. Uber narbige stenosen der trachea. Zentralbl Chir 1886;13:759-60.

4. Sanger PW. Evacuation hospital experience with war wounds and injuries of the chest. Ann Surg 1945;122:147-62.

5. Miura T, Grillo HC. The contribution of the inferior thyroid artery to the blood supply of the human trachea. Surg Gynecol Obstet 1966;123:99-102.

6. Salassa JR, Pearson BW, Payne WS. Gross and microscopic blood supply of the trachea. Ann Thorac Surg 1977;24:100-7.

7. Grillo HC. Surgery of the trachea. Curr Probl Surg 1970:3-59.

8. McClish A, Deslauriers J, Beaulieu M, et al. High flow catheter ventilation during major tracheobronchial reconstruction. J Thorac Cardiovasc Surg 1985;89:508-12.

9. Grillo HC. Reconstruction of the trachea. Experience in 100 consecutive cases. Thorax 1973;28:667-79.

10. Grillo HC, Mathisen DJ. Primary tracheal tumors: treatment and results. Ann Thorac Surg 1990;49:69-77.

11. Mahtabifard A, Fuller CB, McKenna RJ Jr. Video-assisted thoracic surgery sleeve lobectomy: a case series. Ann Thorac Surg 2008;85:S729-32.

12. McKenna RJ Jr, Houck W, Fuller CB. Video-assisted thoracic surgery lobectomy: experience with 1,100 cases. Ann Thorac Surg 2006;81:421-5; discussion 425-6.

13. Gonzalez-Rivas D, Yang Y, Stupnik T, et al. Uniportal videoassisted thoracoscopic bronchovascular, tracheal and carinal sleeve resections. Eur J Cardiothorac Surg 2016;49:16-16.

14. Gonzalez-Rivas D, Fernandez R, Fieira E, et al. Uniportal video-assisted thoracoscopic bronchial sleeve lobectomy: first report. J Thorac Cardiovasc Surg 2013;145:1676-7.

15. Gonzalez-Rivas D, Delgado M, Fieira E, et al. Left lower sleeve lobectomy by uniportal video-assisted thoracoscopic approach. Interact Cardiovasc Thorac Surg 2014;18:237-9.

16. Soultanis KM, Chen Chao M, Chen J, et al. Technique and outcomes of 79 consecutive uniportal video-assisted sleeve lobectomies. Eur J Cardiothorac Surg 2019;56:876-82.

17. Li J, Wang W, Jiang L, et al. Video-assisted thoracic surgery resection and reconstruction of carina and trachea for malignant or benign disease in 12 patients: three centers' experience in China. Ann Thorac Surg 2016;102:295-303.

18. Gonzalez-Rivas D, Yang Y, Sekhniaidze D, et al. Uniportal video-assisted thoracoscopic bronchoplastic and carinal sleeve procedures. J Thorac Dis 2016;8:S210-22.

19. Paul S, Altorki NK, Sheng S, et al. Thoracoscopic lobectomy is associated with lower morbidity than open lobectomy: a propensity-matched analysis from the STS database. J Thorac Cardiovasc Surg 2010;139:366-78.

20. Handy JR, Asaph JW, Douville EC, et al. Does video assisted thoracoscopic lobectomy for lung cancer provide improved functional outcomes compared with open lobectomy? Eur J Cardiothorac Surg 2010;37:451-5.

21. Taioli E, Lee DS, Lesser M, et al. Long-term survival 
in video-assisted thoracoscopic lobectomy vs open lobectomy in lung-cancer patients: a meta-analysis. Eur J Cardiothorac Surg 2013;44:591-7.

22. Zhang Z, Zhang $\mathrm{Y}$, Feng $\mathrm{H}$, et al. Is video-assisted thoracic surgery lobectomy better than thoracotomy for early-stage non-small-cell lung cancer? A systematic review and metaanalysis. Eur J Cardiothorac Surg 2013;44:407-14.

23. Ng CS, Lee TW, Wan S, et al. Thoracotomy is associated with significantly more profound suppression in lymphocytes and natural killer cells than video-assisted thoracic surgery following major lung resections for cancer. J Invest Surg 2005;18:81-8.

24. Detterbeck FC, Lewis SZ, Diekemper R, et al. Executive Summary: Diagnosis and management of lung cancer, 3rd ed: American College of Chest Physicians evidence-based clinical practice guidelines. Chest 2013;143:7S-37S.

25. Gonzalez D, Paradela M, Garcia J, et al. Single-port videoassisted thoracoscopic lobectomy. Interact Cardiovasc Thorac Surg 2011;12:514-5.

26. Liu Z, Yang R, Shao F. Comparison of postoperative pain and recovery between single-port and two-port thoracoscopic lobectomy for lung cancer. Thorac Cardiovasc Surg 2019;67:142-6.

27. Regnard JF, Perrotin C, Giovannetti R, et al. Resection of tumors with carinal involvement: technical aspects, results and prognostic factors. Ann Thorac Surg 2005;80:1841-6.

28. Brunelli A, Kim A, Berger K, et al. Physiologic evaluation of the patient with lung cancer being considered for resectional surgery. Chest 2013;143:e166S-90S.

29. Broussard B, Mathisen DJ. Tracheal release maneuvers. Ann Cardiothorac Surg 2018;7:293-8.

30. Bhattacharyya N. Contemporary staging and prognosis for primary tracheal malignancies: a population-based analysis. Otolaryngol Head Neck Surg 2004;131:639-42.

31. Gaissert HA, Grillo HC, Shadmehr MB, et al. Longterm survival after resection of primary adenoid cystic and squamous cell carcinoma of the trachea and carina. Ann Thorac Surg 2004;78:1889-96.

32. Grillo HC. Carinal Reconstruction. In: Grillo HC. Surgery of the trachea and bronchi. Raleigh: PMPH USA, 2004:599.

33. Udelsman B, Mathisen DJ, Ott HC. A reassessment of tracheal substitutes-a systematic review. Ann Cardiothorac Surg 2018;7:175-82.

34. Grillo HC. Reconstruction of the Lower Trachea (Transthoracic) and Procedures for Extended Resection. In: Grillo HC. Surgery of the trachea and bronchi. Raleigh: PMPH USA, 2014:587.

35. Wright CD, Grillo HC, Wain JC, et al. Anastomotic complications after tracheal resection: prognostic factors and management. J Thorac Cardiovasc Surg 2004;128:731-9.

36. Grillo HC, Dignan EF, Miura T. Extensive resection and reconstruction of the mediastinal trachea without prosthesis or graft: an anatomical study in man. J Thorac Cardiovasc Surg 1964;48:741-9.

37. Lanuti M, Mathisen D. Carinal resection. Thorac Surg Clin 2004;14:199-209.

38. de Perrot M, Fadel E, Mercier O, et al. Long-term results after carinal resection for carcinoma: does the benefit warrant the risk? J Thorac Cardiovasc Surg 2006;131:81-9.

39. Grillo HC. Main and lobar bronchoplasty, In: Grillo HC. Surgery of the trachea and bronchi. Raleigh: PMPH USA, 2014:622.

40. Palade E, Holdt H. Passlick B. Bronchus anastomosis after sleeve resection for lung cancer: does the suture technique have an impact on postoperative complication rate? Interact Cardiovasc Thorac Surg 2015;20:798-804.

41. Porhanov VA, Poliakov IS, Selvaschuk AP, et al. Indications and results of sleeve carinal resection. Eur J Cardiothorac Surg 2002;22:685-94.

42. Frist WH, Mathisen DJ, Hilgenberg AD, Grillo HC. Bronchial sleeve resection with and without pulmonary resection. J Thorac Cardiovasc Surg 1987;93:350-7.

43. Kutlu CA, Goldstraw P. Tracheobronchial sleeve resection with the use of a continuous anastomosis: results of one hundred consecutive cases. J Thorac Cardiovasc Surg 1999;117:1112-7.

44. Grillo HC. Anesthesia for Tracheal Surgery. In: Grillo HC. Surgery of the trachea and bronchi. Raleigh: PMPH USA, 2014:643.

45. Jiang L, Liu J, Gonzalez-Rivas D, et al. Thoracoscopic surgery for tracheal and carinal resection and reconstruction under spontaneous ventilation. J Thorac Cardiovasc Surg 2018;155:2746-54.

46. Horita K, Itoh T, Furukawa K, et al. Carinal reconstruction under veno-venous bypass using a percutaneous cardiopulmonary bypass system. Thorac Cardiovasc Surg 1996;44:46-9.

47. Lyscov A, Obukhova T, Ryabova V, et al. Double-sleeve and carinal resections using the uniportal VATS technique: a single centre experience. J Thorac Dis 2016;8:S235-41.

Cite this article as: Gonzalez-Rivas D, Soultanis KM, Garcia A, Yang K, Qing Y, Yie L, Zhao G, Chen A, Huang Y, Li G, Jiang G. Uniportal video-assisted thoracoscopic lung sparing tracheo-bronchial and carinal sleeve resections. J Thorac Dis 2020;12(10):6198-6209. doi: 10.21037/jtd.2020.04.05 